\title{
Sodium tanshinone IIA sulfonate attenuates the transforming growth factor- $\beta 1$-induced differentiation of atrial fibroblasts into myofibroblasts in vitro
}

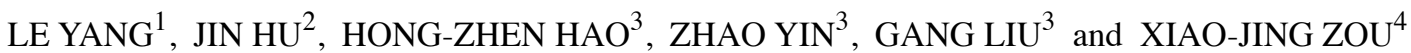 \\ Departments of ${ }^{1}$ Emergency Medicine, ${ }^{2}$ Otolaryngology, Head and Neck Surgery, Tongji Hospital, \\ and ${ }^{3}$ Department of Pharmacology, Tongji Medical College, Huazhong University of Science and Technology, \\ Wuhan, Hubei 430030; ${ }^{4}$ Department of Anesthesiology and Critical Care Medicine, Laboratory of Anesthesiology \\ and Critical Care Medicine, Union Hospital, Tongji Medical College, Huazhong University \\ of Science and Technology, Wuhan, Hubei 430022, P.R. China
}

Received November 8, 2014; Accepted January 15, 2015

DOI: $10.3892 /$ ijmm.2015.2087

\begin{abstract}
The differentiation of atrial fibroblasts into myofibroblasts is a critical event in atrial fibrosis. One of the most important factors in atrial fibroblast differentiation is transforming growth factor- $\beta 1$ (TGF- $\beta 1$ ). Accumulating evidence indicates that sodium tanshinone IIA sulfonate (STS) possesses antifibrotic properties. In this study, we therefore investigated whether STS attenuates the TGF- $\beta 1$-induced differentiation of atrial fibroblasts. TGF- $\beta 1$ enhanced collagen production, collagen synthesis and the expression of collagen type I and III, as shown by hydroxyproline assay, collagen synthesis assay and western blot analysis, respectively. In addition, as shown by immunohistochemistry and western blot analysis, TGF- $\beta 1$ enhanced the expression of $\alpha$-smooth muscle actin ( $\alpha$-SMA), which is the hallmark of myofibroblast differentiation. These responses were attenuated by treatment with STS. In addition, STS suppressed the TGF- $\beta 1$-induced expression of phosphorylated (p)Smad/pSmad3 expression and nuclear translocation. Furthermore, STS supressed extracellular signal-regulated kinase (ERK) phosphorylation. In conclusion, the current study demonstrates that STS exerts antifibrotic effects by modulating atrial fibroblast differentiation through ERK phosphorylation and the Smad pathway.
\end{abstract}

\section{Introduction}

Atrial fibrillation (AF) is the most common arrhythmia observed in humans and accounts for significant morbidity and mortality

Correspondence to: Dr Xiao-Jing Zou, Department of Anesthesiology and Critical Care Medicine, Laboratory of Anesthesiology and Critical Care Medicine, Union Hospital, Tongji Medical College, Huazhong University of Science and Technology, 1277 Jiefang Avenue, Wuhan, Hubei 430022, P.R. China

E-mail:xjzou66@yahoo.com

Key words: tanshinone IIA, transforming growth factor- $\beta 1$, atrial fibroblast, differentiation rates. It is a mechanistically complex arrhythmia for which available therapies remain suboptimal (1). Atrial fibrosis is a common characteristic of clinical AF and is associated with AF in a variety of experimental paradigms (2). Increased collagen deposition has been documented in patients with lone AF compared with the control subjects with normal sinus rhythm (3). It therefore stands to reason that future therapies for AF should be aimed at reversing or delaying the fibrotic process (4).

Atrial fibroblasts are a quantitatively and qualitatively important cell type of the heart. Extensive evidence indicates that atrial fibrosis, involving fibroblast differentiation and increased fibroblast-derived ECM protein expression, plays an important role in AF (5). Animal studies have demonstrated the importance of transforming growth factor- $\beta 1$ (TGF- $\beta 1$ ) in atrial fibrosis. The inhibition of TGF- $\beta 1$ prevents atrial fibrosis, atrial conduction abnormalities and vulnerability to AF (6). TGF- $\beta 1$ is fibrogenic cytokine that plays a key role in the differentiation of fibroblasts into myofibroblasts, which express $\alpha$-smooth muscle actin ( $\alpha$-SMA) and produce more collagen than fibroblasts (7). The primary TGF- $\beta 1$ signal transduction pathway is the conserved Smad pathway (8). Receptor-regulated Smads (R-Smads, including Smad2 and Smad3) and inhibitory-Smad7 (I-Smad7) have been well characterized as key downstream effectors of TGF- $\beta 1$ signaling (9). The TGF- $\beta /$ Smad pathway is recognized as a traditional signaling pathway that initiates the activation of cardiac fibroblasts $(10,11)$. In addition, previous studies have shown that TGF- $\beta /$ Smad signaling is tightly controlled by mitogen-activated protein (MAP) kinase signaling cascades, particularly extracellular signal-regulated kinase 1/2 (ERK1/2) $(12,13)$.

Sodium tanshinone IIA sulfonate (STS) is a derivative of tanshinone IIA, which is a lipid-soluble pharmacologically active component isolated from the rhizome of the Chinese herb, Salvia miltiorrhiza, a well-known traditional Chinese medicine. Tanshinone IIA has been shown to possess a variety of biological activities in the cardiovascular system, such as antioxidant, anticoagulant, anti-atherosclerotic, anti-apoptotic and antihypertrophic activities (14-17). Tanshinone IIA has also been shown to possess anti-fibrotic activities $(18,19)$. In 
the present study, we explored the effects and mechanisms of action of STS on the TGF- $\beta 1$-induced collagen expression and differentiation of atrial fibroblasts into myofibroblasts in vitro, demonstrating the potential application of STS in clinical practice for the treatment of atrial fibrosis and AF.

\section{Materials and methods}

Materials. Week-old neonatal Wistar rats were obtained from the Experimental Animal Center of Tongji Medical College, Grate II. The present study complied with the National Institutes of Health Guidelines on the Use of Laboratory Animals and was approved by the Animal Research Committee of Tongji Medical College, Huazhong University of Science and Technology (Wuhan, China). STS (99.5\%) was obtained from the Research Center of Traditional Chinese Medicine (Wuhan, China) and was dissolved in phosphate-buffered saline (PBS). TGF- $\beta 1$ was obtained from Sigma-Aldrich (St. Louis, MO, USA). PD98059 (ERK inhibitor) was purchased from Calbiochem (San Diego, CA, USA). Antibodies, such as those for collagen type I (no. sc-8784)/III (no. sc-8781), $\alpha$-SMA (no. sc-32251), phosphorylated (p)Smad2 (no. sc-101801) and pERK1/2 (no. sc-101761), were purchased from Santa Cruz Biotechnology, Inc. (Dallas, TX, USA). All other chemicals used were of the highest quality available commercially. The molecular structure of STS is illustrated in Fig. 1.

Cell culture. Primary cultured atrial fibroblasts were prepared as previously described (20). Briefly, the atria from week-old neonatal Wistar rats were separated and minced with scissors into $2-3-\mathrm{mm}^{3}$ fragments in ice-cold balanced salt solution. The fragments were then digested by 4-6 cycles ( 15 min each) of incubation with $0.125 \%$ trypsin. At the end of each cycle, the supernatant was stored on ice after the addition of fetal bovine serum (FBS) to neutralize trypsin. The dissociated cells were collected by centrifugation at $1,000 \mathrm{x} \mathrm{g}$ for $10 \mathrm{~min}$, followed by resuspension in Dulbecco's modified Eagle's medium (DMEM) supplemented with 10\% FBS. Thereafter, the cells were incubated in a humidified atmosphere of $5 \% \mathrm{CO}_{2}$ at $37^{\circ} \mathrm{C}$. After $1 \mathrm{~h}$ of incubation, the atrial fibroblasts attached to the dishes, whereas cardiomyocytes remaining in the medium were discarded. The attached atrial fibroblasts were further cultured to confluence and then passaged at a 1:3 dilution. The first and second passages fibroblasts were used for all the experiments.

Collagen production assay. The collagen content in the fibroblast cultures was measured by hydroxyproline assay as previously described (21). Briefly, the fibroblasts were made quiescent by culture in serum-free DMEM for $24 \mathrm{~h}$. The quiescent cells were then pre-treated with STS $(3,10$ and $30 \mu \mathrm{M})$ for $30 \mathrm{~min}$ and then exposed to TGF- $\beta 1(5 \mathrm{ng} / \mathrm{ml})$ for $48 \mathrm{~h}$. The protein content in the cell lysates the was measured using Coomassie reagent (Santa Cruz Biotechnology, Inc.).

Collagen synthesis assay. Collagen synthesis was evaluated by measuring $\left[{ }^{3} \mathrm{H}\right]$ proline incorporation as previously described (22). In brief, the fibroblasts were made quiescent by culture in serum-free DMEM for $24 \mathrm{~h}$. Subsequently, these cells were treated with STS $(3,10$ and $30 \mu \mathrm{M})$ for $30 \mathrm{~min}$ prior to stimulation with $5 \mathrm{ng} / \mathrm{ml}$ TGF- $\beta 1$ for $24 \mathrm{~h}$. Collagen

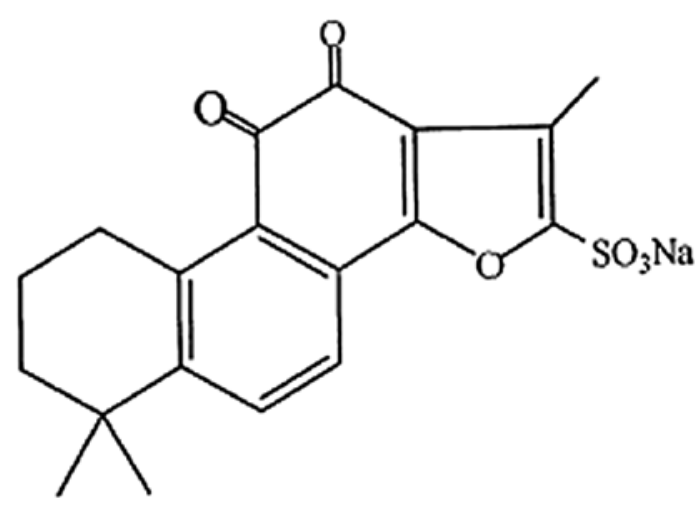

Figure 1. The molecular structure of sodium tanshinone IIA sulfonate (STS)

synthesis was assessed by the addition of $1 \mu \mathrm{Ci} / \mathrm{mL}\left[{ }^{3} \mathrm{H}\right]$ proline (Amersham Biosciences, Piscataway, NJ, USA) for $6 \mathrm{~h}$. The cells were washed with PBS and ice-cold 10\% trichloroacetic acid. The cells were then solubilized and the cell extracts were analyzed in a liquid scintillation counter [1450; PerkinElmer Enspire (China) Co., Ltd., Shanghai, China].

Immunocytochemistry/ $\alpha-S M A$ staining. Following TGF- $\beta 1$ $(5 \mathrm{ng} / \mathrm{ml})$ stimulation for $24 \mathrm{~h}$ with or without the presence of STS $(10 \mu \mathrm{M})$, the cells were washed with PBS, fixed with $4 \%$ paraformaldehyde in PBS for $20 \mathrm{~min}$, and then permeablized with $0.1 \%$ Triton X-100/PBS for $20 \mathrm{~min}$. After the cells were incubated with a blocker ( $3 \%$ bovine serum) for $30 \mathrm{~min}$, the specific anti$\alpha$-SMA antibody was added. The cells were visualized under a fluorescence microscope (Olympus, Tokyo, Japan).

Nuclear, cytoplasmic and total protein extract preparation and western blot analysis. Nuclear and cytoplasmic extracts of fibroblasts were prepared as previously described (23). Nuclear proteins and cytoplasmic proteins were extracted using a nuclear extraction kit (Panomics, Santa Clara, CA, USA) following the manufacturer's instructions and stored at $-20^{\circ} \mathrm{C}$ until used in western blot analysis. Proteins (30 $\mu \mathrm{g})$ were separated on $4-15 \%$ gradient polyacrylamide gels, transferred onto PVDF membranes and probed with the following antibodies: mouse monoclonal anti- $\alpha$-SMA (no. A2547), rabbit anti-Smad2 (no. SAB4501806), rabbit anti-phospho Smad2 (pSmad2; no. sc-101801), rabbit anti-Smad3 (no. SAB4200342), rabbit antiphospho Smad3 (pSmad3; no. SAB4504210), rabbit anti-Smad7 (no. SAB4200345), rabbit anti-phospho-ERK1/2 (no. sc-101761), and rabbit anti-total-ERK1/2 (no. sc-292838). All blots were exposed for optimal lengths of time for visualization.

Statistical analysis. The results are expressed as the means \pm SD. Statistical significance was determined by one-way ANOVA. The differences were considered statistically significant at a value of $\mathrm{P}<0.05$.

\section{Results}

Effects of STS on the TGF- $\beta 1$-induced collagen production and synthesis. TGF- $\beta 1(5 \mathrm{ng} / \mathrm{ml})$ significantly enhanced collagen production, which was attenuated by treatment with 

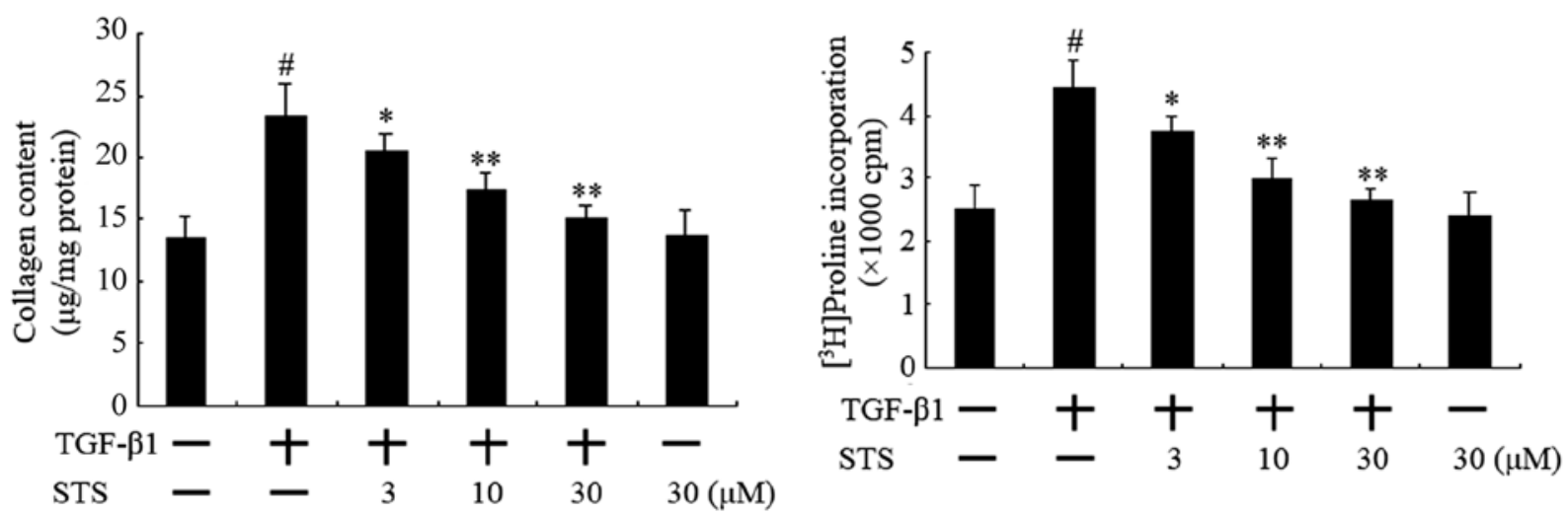

Figure 2. Effects of sodium tanshinone IIA sulfonate (STS) on transforming growth factor- $\beta 1$ (TGF- $\beta 1$ )-induced collagen production and collagen synthesis. Atrial fibroblasts were treated with STS $(3,10$ and $30 \mu \mathrm{M})$ for $30 \mathrm{~min}$ prior to stimulation with $5 \mathrm{ng} / \mathrm{ml} \mathrm{TGF}-\beta 1$. After $24 \mathrm{~h}$, the collagen content of fibroblast cultures was measured by hydroxyproline assay, and collagen synthesis was determined with the $\left[{ }^{3} \mathrm{H}\right]$ proline incorporation. The experiments were repeated 4 times with reproducible results. ${ }^{\#} \mathrm{P}<0.01$ vs. controls; ${ }^{*} \mathrm{P}<0.05$ and ${ }^{* *} \mathrm{P}<0.01$ vs. TGF- $\beta 1$-treated group.
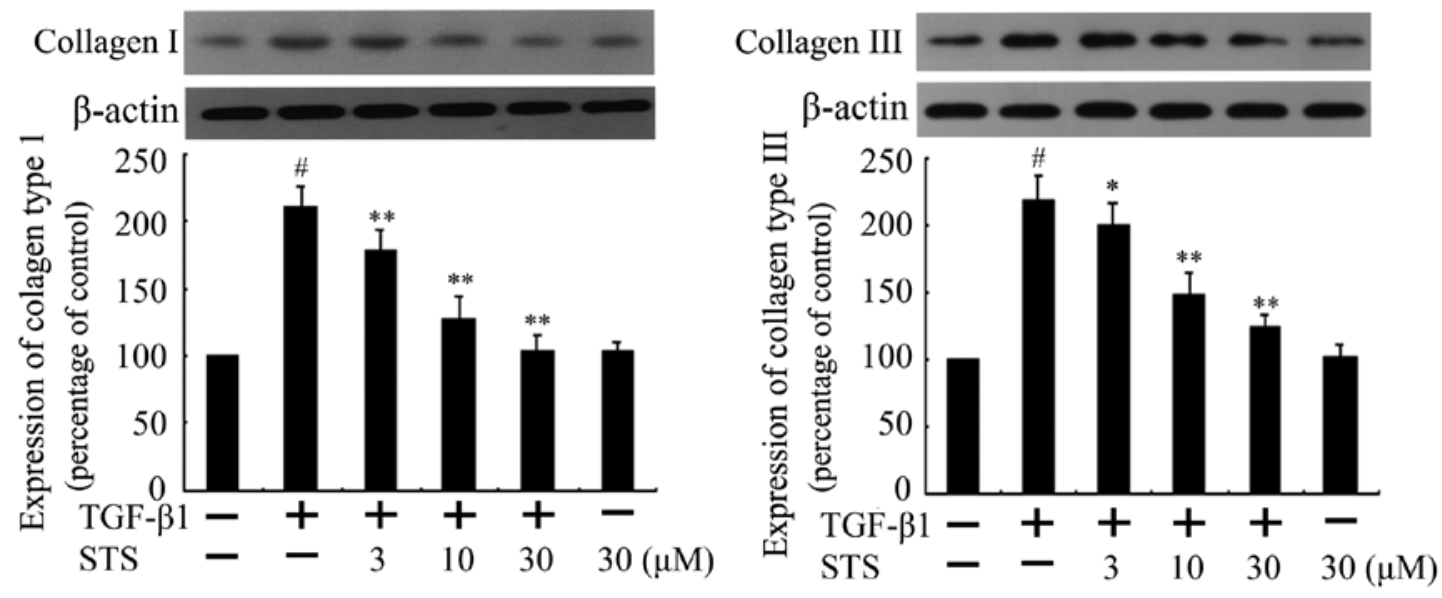

Figure 3. Effects of sodium tanshinone IIA sulfonate (STS) on the transforming growth factor- $\beta 1$ (TGF- $\beta 1$ )-induced expression of collagen type I and III. Atrial fibroblasts were treated with STS $(3,10$ and $30 \mu \mathrm{M})$ for $30 \mathrm{~min}$ prior to stimulation with $5 \mathrm{ng} / \mathrm{ml}$ TGF- $\beta 1$. After $24 \mathrm{~h}$, collagen type I and III expression was measured by western blot analysis. The experiments were repeated 4 times with reproducible results. ${ }^{\prime \prime} \mathrm{P}<0.01$ vs. controls; ${ }^{*} \mathrm{P}<0.05$ and ${ }^{* * *} \mathrm{P}<0.01$ vs. TGF- $\beta 1-$ treated group.

STS (3, 10 and $30 \mu \mathrm{M})$ (Fig. 2). Consistent with the increase in collagen production, TGF- $\beta 1$ induced a significant increase in the rate of $\left[{ }^{3} \mathrm{H}\right]$ proline incorporation, which was also markedly suppressed by treatment with STS. However, STS alone had no effect on collagen production and synthesis.

Effects of STS on the TGF- $\beta 1$-induced expression of collagen type I and III. Type I and III collagen constitute two-thirds of cardiac collagen. TGF- $\beta 1$ significantly enhanced the expression of collagen type I and III; this effect was attenuated by treatment with STS (3, 10 and $30 \mu \mathrm{M})$ (Fig. 3). Consistent with the increase in collagen expression, TGF- $\beta 1(5 \mathrm{ng} / \mathrm{ml})$ induced a significant increase in the rate of $\left[{ }^{3} \mathrm{H}\right]$ proline incorporation, which was also significantly suppresswed by treatment with STS. Therefore, STS attenuates TGF- $\beta 1$-induced fibrotic responses in atrial fibroblasts.

Effects of STS on the TGF- $\beta 1$-induced expression of $\alpha$-SMA. The expression of $\alpha$-SMA is a hallmark of myofibroblast differentiation (24-26). Therefore, we assessed the expression levels of $\alpha$-SMA $24 \mathrm{~h}$ following treatment with TGF- $\beta 1$ in the presence or absence of STS. TGF- $\beta 1$ stimulation increased the intensity and organization of $\alpha$-SMA, which was markedly suppressed by treatment with $10 \mu \mathrm{M}$ STS (Fig. 4). Moveover, western blot analysis revealed that treatment with $5 \mathrm{ng} / \mathrm{ml}$ TGF- $\beta 1$ significantly enhanced the expression of $\alpha$-SMA, which was suppressed by STS.

Effects of STS on the TGF- $\beta 1$-induced phosphorylation of Smad2, Smad 3 and ERK1/2, and Smad7. TGF- $\beta 1$ signals were followed by the activation of cytoplasmic effectors, such as Smad proteins. The phosphorylation of $S \operatorname{mad} 2 / \mathrm{Smad} 3$ and the subsequent translocation of $\mathrm{Smad} 2 / \mathrm{Smad} 3$ to the nucleus are critical steps in cell signaling through this pathway. Inhibitory Smads, such as Smad7, antagonize these processes $(12,13,27)$. Western blot analysis revealed that TGF- $\beta 1$ significantly increased the phosphorylation of Smad2 and Smad3. STS significantly blocked the TGF- $\beta 1$-induced expression of pSmad 2 and pSmad3 (Fig. 5). However, STS did not exert any significant effect on Smad7 expression (Fig. 5). 


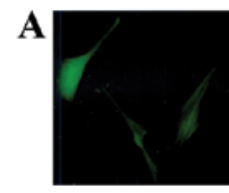

Control

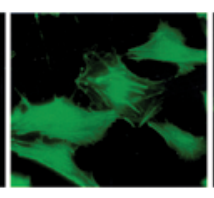

TGF-B1

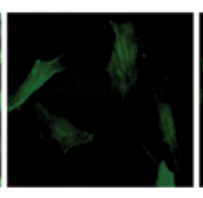

TGF-B1+STS

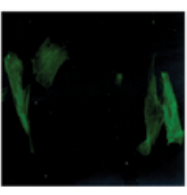

STS
B
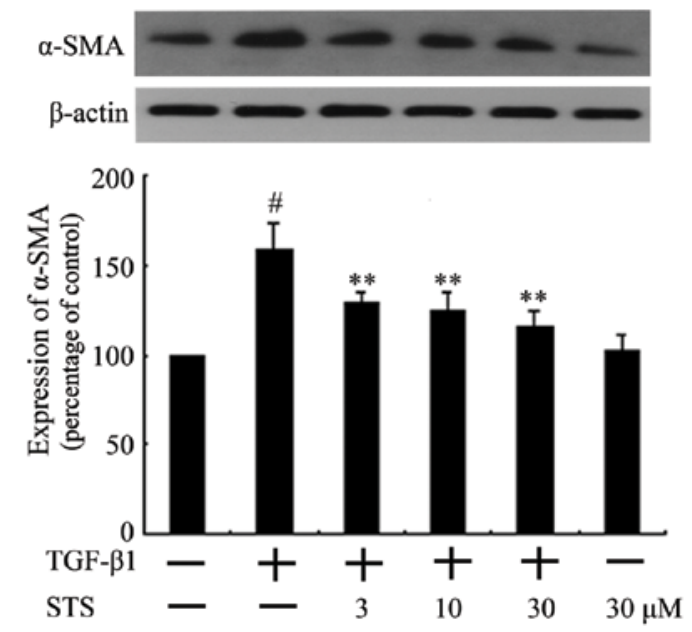

Figure 4. Effects of sodium tanshinone IIA sulfonate (STS) on the transforming growth factor- $\beta 1$ (TGF- $\beta 1$ )-induced expression of $\alpha$-smooth muscle actin ( $\alpha$-SMA). Atrial fibroblasts were treated with STS for 30 min prior to stimulation with $5 \mathrm{ng} / \mathrm{ml}$ TGF- $\beta 1$. For the immunocytochemical staining of $\alpha$-SMA, atrial fibroblasts were pre-treated with $5 \mathrm{ng} / \mathrm{ml}$ TGF- $\beta 1$ in the presence and absence of $10 \mu \mathrm{M}$ STS (magnification, $\mathrm{x} 400$ ). $\alpha$-SMA expression was determined by western blot analysis. ${ }^{~} \mathrm{P}<0.01$ vs. controls; ${ }^{* *} \mathrm{P}<0.01$ vs. TGF- $\beta 1$-treated group.

In addition, a recent study revealed that the pharmacological inhibition of ERKs blocked the gene expression of TGF- $\beta 1$ stimulated collagen in cardiac fibroblasts (28). We also found that treatment with PD98059 (an ERK inhibitor) at $25 \mu \mathrm{M}$ decreased the TGF- $\beta 1$-induced expression of $\alpha$-SMA (Fig. 6). Another study demonstrated that the activation of ERK was necessary for both myofibroblast differentiation and ECM synthesis induced by TGF- $\beta 1$ in the cardiac fibroblasts of neonatal rats (29). Therefore, we examined whether ERK1/2 activation is induced by stimulation with TGF- $\beta 1$ in atrial fibroblasts and whether STS has any effect on this process. The phosphorylation of ERK1/2 was increased in the TGF- $\beta 1$-stimulated fibroblasts and was significantly suppressed by treatment with STS (Fig. 6).

Effects of STS on the TGF- $\beta 1$-induced nuclear translocation of pSmad2 and pSmad3. To determine whether the inhibition of TGF- $\beta 1$-induced expression of $\alpha$-SMA by STS is dependent on events downstream of the phosphorylation of Smad2 and Smad3, we examined the effects of STS on the TGF- $\beta 1$ induced nuclear translocation of $\mathrm{pSmad} 2$ and $\mathrm{pSmad} 3$ in atrial fibroblasts. Protein samples were prepared from cytoplasmic and nuclear fractions form the different treatment groups. Treatment with TGF- $\beta 1$ resulted in a strong accumulation of pSmad 2 and pSmad 3 in the nucleus (Fig. 7). The above effects were significantly suppressed by treatment with STS.

\section{Discussion}

AF results in undirected wavefronts in the atria (30). This leads to functionally inadequate atrial contractions, which slow the blood flow velocity in the atria, thus increasing atrial blood load and preventing optimal ventricular filling (31). Atrial fibrosis is a common pathological condition observed in AF, particularly in long-standing AF. Atrial fibrosis is also observed in AF associated with underlying structural disease, including various forms of cardiomyopathies and mitral valve diseases $(32,33)$.

Atrial fibroblasts are the major source of collagen in the atrial endocardium and behave differently than ventricular fibroblasts over a range of in vitro and in vivo paradigms. Atrial fibroblasts show enhanced reactivity, which may explain greater atrial fibrotic responses $(5,34)$. As they have the form of thick fiber with a high tensile strength, collagen type I and III contents are considered major determinants of myocardial stiffness (35-37). TGF- $\beta 1$ is a key fibrogenic cytokine both in vitro and in vivo (38). As previously demonstrated, in TGF- $\beta 1$-deficient mice and rats treated with anti-TGF- $\beta 1$ antibodies, cardiac fibrosis is blunted (39). Many other fibrogenic factors, such as angiotensin II, and mechanical stretch and oxidative stress increase TGF- $\beta 1$ secretion in fibroblasts (40). The number of myofibroblasts has been shown to be increased in rats with cardiac fibrosis resulting from hypertension or myocardial infarction (41). Activated myofibroblasts are considered the major source of ECM components, such as collagen type I and III (42). Hence, the conversion of fibroblasts into myofibroblasts by TGF- $\beta 1$ is an important mechanism in the development of cardiac fibrosis (43).

Tanshinone IIA is most abundant and structurally representative of the tanshinones of Salvia miltiorrhiza (44). STS has also shown to be a promising drug that reduces cardiac remodeling by suppressing cardiomyocyte hypertrophy (45). Moreover, STS has been shown to possess antifibrotic activities in vivo $(18,19)$. Tanshinone IIA has also been reported to have an antiarrhythmic effect, decreasing the duration of lethal arrhythmias and reducing the incidence of ventricular tachycardia and/or ventricular fibrillation (VT/VF) in rats with myocardial infarction (46). Nevertheless, to date, little is known of the cellular and molecular mechanisms responsible for the STS-mediated antifibrotic effects in atrial fibroblasts following stimulation with TGF- $\beta 1$. In the present study, we attempted to explore the effects and mechanisms of action of STS on the TGF- $\beta 1$-induced differentiation of cultured atrial fibroblasts into myofibroblasts.

In the present in vitro study, we demonstrated that TGF- $\beta 1$ increased collagen production and synthesis. Furthermore, TGF- $\beta 1$ increased the expression of collagen type I and III in atrial fibroblasts. These results were consistent with those of a recent study (47). The above effects were significantly suppressed by treatment with STS. Therefore, STS supresses the TGF- $\beta 1$-induced expression of collagen type I and III through the attenuation of collagen synthesis. To further determine the effects of STS on the TGF- $\beta 1$-induced differentiation of atrial fibroblasts into myofibroblasts, immunofluorescence staining for $\alpha$-SMA was used to detect $\alpha$-SMA expression and the morphological changes of atrial fibroblasts. Compared with the control group, the atrial fibroblasts stimulated with $5 \mathrm{ng} / \mathrm{ml}$ of TGF- $\beta 1$ showed bright staining for $\alpha$-SMA, the appearance of stress fibers and a larger cell size. These effects be were suppressed by treatment with STS (Fig. 4), which indicates that STS has obvious antifibrotic effects. 

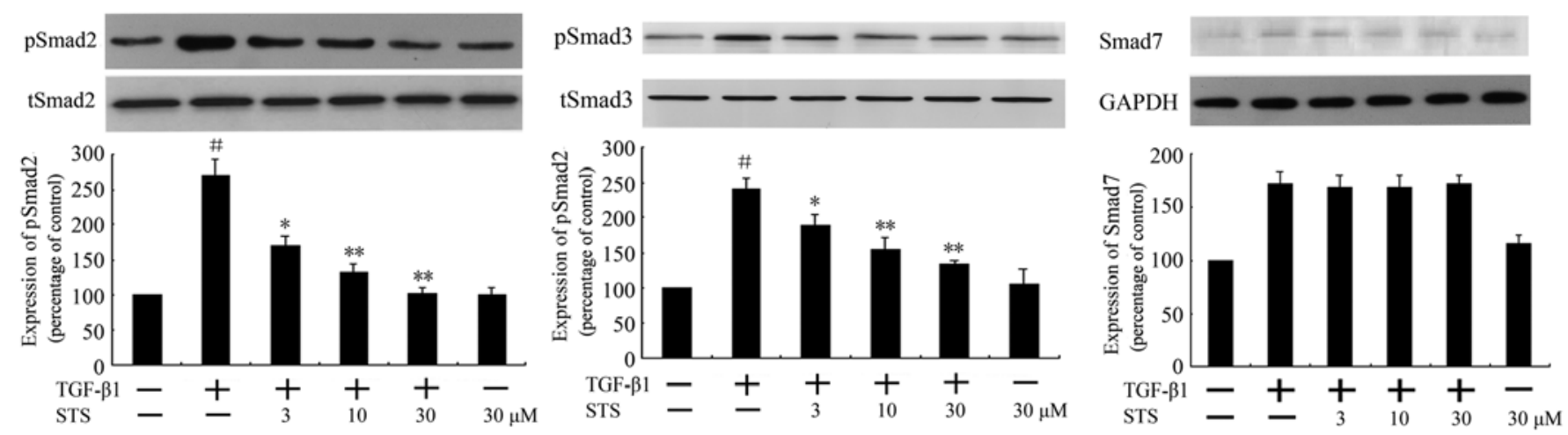

Figure 5. Effects of sodium tanshinone IIA sulfonate (STS) on the transforming growth factor- $\beta 1$ (TGF- $\beta 1$ )-induced phosphorylation of Smad2, Smad3 and Smad7 expression. Atrial fibroblasts were treated with STS $(3,10$ and $30 \mu \mathrm{M})$ for $30 \mathrm{~min}$ prior to stimulation with $5 \mathrm{ng} / \mathrm{ml} \mathrm{TGF}-\beta 1$ for $30 \mathrm{~min}$. The expression of pSmad2, pSmad3 and Smad7 was measured by western blot analysis. The experiments were repeated 4 times with reproducible results. ${ }^{\# P}<0.01$ vs. controls; ${ }^{*} \mathrm{P}<0.05$ and ${ }^{* *} \mathrm{P}<0.01$ vs. TGF- $\beta 1$-treated group.
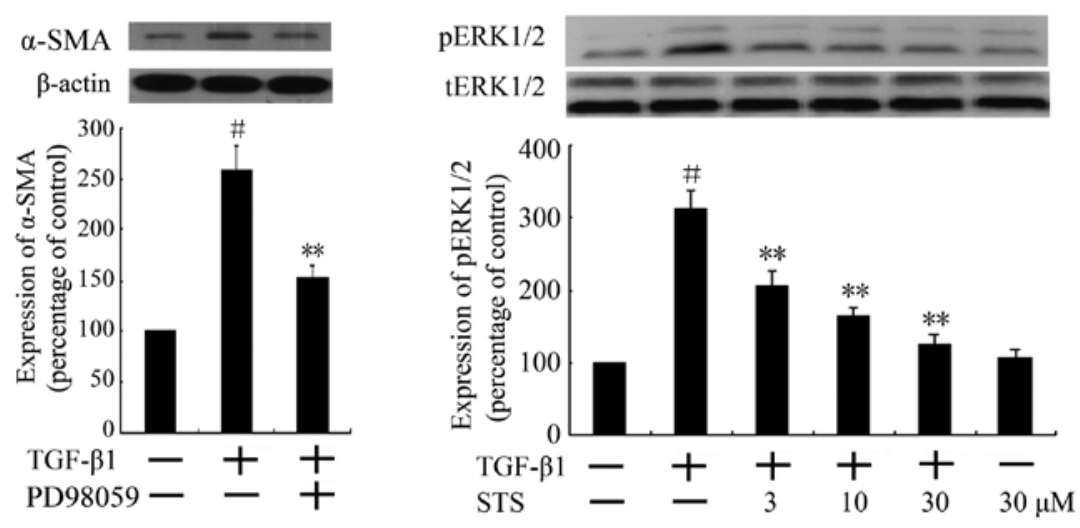

Figure 6. Effects of sodium tanshinone IIA sulfonate (STS) on the transforming growth factor- $\beta 1$ (TGF- $\beta 1$ )-induced phosphorylation of ERK1/2. Atrial fibroblasts were treated with STS $(3,10$ and $30 \mu \mathrm{M})$ for 30 min prior to stimulation with $5 \mathrm{ng} / \mathrm{ml} \mathrm{TGF-} \beta 1$ for $30 \mathrm{~min}$. The expression of phosphorylated ERK1/2 and $\alpha$-smooth muscle actin $\left(\alpha\right.$-SMA) was measured by western blot analysis. The experiments were repeated 4 times with reproducible results. ${ }^{\prime \prime} \mathrm{P}<0.01 \mathrm{vs.}$ controls; ${ }^{* *} \mathrm{P}<0.01$ vs. TGF- $\beta 1$-treated group.
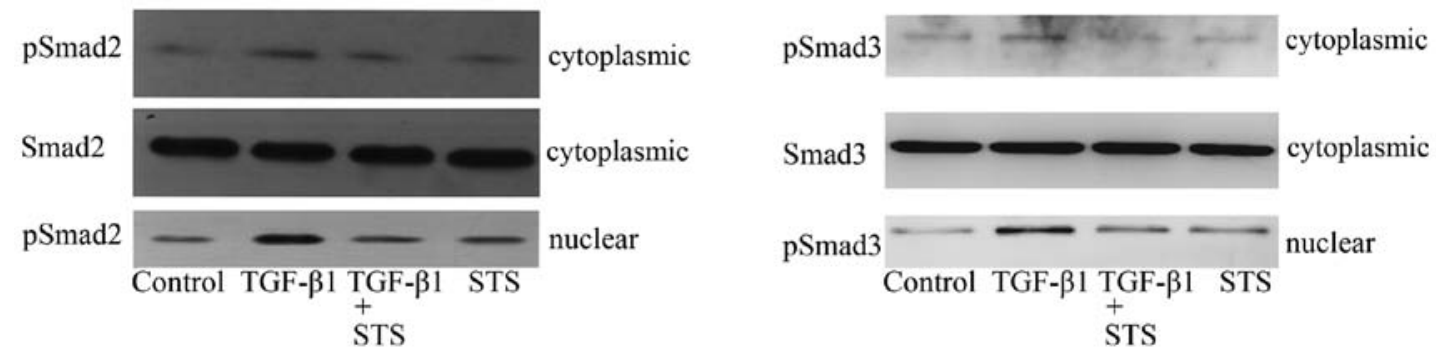

Figure 7. Representative western blot showing that sodium tanshinone IIA sulfonate (STS) inhibits the transforming growth factor- $\beta 1$ (TGF- $\beta 1$ )-induced nuclear translocation of phosphorylated Smad and Smad3. Atrial fibroblasts were treated with STS $(10 \mu \mathrm{M})$ for 30 min prior to stimulation with $5 \mathrm{ng} / \mathrm{ml} \mathrm{TGF-} \beta 1$ for an additional $1 \mathrm{~h}$. Protein samples were prepared from cytoplasmic and nuclear fractions of the different groups.

The biological effects of TGF- $\beta 1$ are mediated through a complex of type II and I receptors characterized as serine-threonine kinases that transduce intracellular signals through both Smad and non-Smad pathways. The activated receptor complex phosphorylates R-Smads, such as Smad2 and Smad3, which form homomeric and heteromeric complexes with a co-Smad (Smad4). These active Smad complexes enter the nucleus, where they bind to target genes and directly regulate their transcription $(9,48)$. Thus, the phosphorylation of R-Smads followed by translocation to the nucleus is a critical step in the activation of TGF- $\beta 1$ signaling. Indeed, as previously demonstrated, the transfection of atrial fibroblasts with a silencing RNA against Smad2 blocked not only the phosphorylation of Smad2, but also the differentiation of fibroblasts into myofibroblasts in response to TGF- $\beta 1$ (49). As expected, our results revealed that pSmad 2 and pSmad 3 expression was significantly increased in the TGF- $\beta 1$-treated cells compared with the controls, and that this effect was significantly inhibited by treatment with STS. 
Moreover, STS suppressed the TGF- $\beta 1$-induced nuclear translocation of pSmad2 and pSmad3 (Fig. 7). Smad7 acts as a negative regulator for both TGF- $\beta /$ Smad signaling pathways (50). These results suggest that STS suppresses the differentiation of atrial fibroblasts into myofibroblasts and collagen synthesis in part through the inhibition of Smad2 and Smad3 phosphorylation.

TGF- $\beta 1 /$ Smad signaling is tightly controlled by the MAP kinase signaling cascades, particularly ERK1/2, in many cell types $(51,52)$. Thus, whereas the ERK1/2 cascade may directly regulate collagen gene transcription, it may also enhance Smad-dependent signaling in atrial fibroblasts in such a manner that the inhibition of ERK signaling also attenuates Smad signaling. Certain studies have reported a positive correlation between TGF- $\beta 1$ and the ERK signaling pathway $(53,54)$. In the present study, we determined the importance of ERK1/2 in TGF- $\beta 1$-induced atrial fibrosis. TGF- $\beta 1$ increased the expression of $\alpha$-SMA, and this effect was decreased by treatment with PD98059 (an ERK inhibitor, $25 \mu \mathrm{M}$ ) (Fig. 6). Moreover, TGF- $\beta 1$ enhanced the ERK1/2 phosphorylation of ERK1/2, which was depressed by STS. Therefore, the inhibition of ERK $1 / 2$ phosphorylation may be one of the mechanisms involved in the downregulation of the TGF- $\beta 1 /$ Smad pathway by STS.

In conclusion, the present study provides important new insight into the molecular mechanisms of action of STS in atrial fibroblasts. Our data also demonstrate that STS exerts antifibrotic effects by modulating the differentiation of atrial fibroblasts through ERK1/2 phosphorylation and the $\operatorname{Smad} 2 / 3$ pathway. Although the precise mechanisms through which STS attenuates the development of atrial fibrosis remain to be further clarified, the understanding of the pharmacological effects of STS on atrial fibroblasts may contribute to the use of STS in the treatment of patients suffering from cardiovascular diseases.

\section{Acknowledgements}

This study was supported by a grant (81102691) from the National Natural Science Foundation of China. The authors wish to express their thanks to Dr Zhao for his generous technical support.

\section{References}

1. Nattel S and Opie LH: Controversies in atrial fibrillation. Lancet 367: 262-272, 2006.

2. Velagapudi P, Turagam MK, Leal MA and Kocheril AG: Atrial fibrosis: a risk stratifier for atrial fibrillation. Expert Rev Cardiovasc Ther 11: 155-160, 2013.

3. Garg A and Akoum N: Atrial fibrillation and heart failure: beyond the heart rate. Curr Opin Cardiol 28: 332-336, 2013.

4. Jalife J: Mechanisms of persistent atrial fibrillation. Curr Opin Cardiol 29: 20-27, 2014.

5. Yue L, Xie J and Nattel S: Molecular determinants of cardiac fibroblast electrical function and therapeutic implications for atrial fibrillation. Cardiovasc Res 89: 744-753, 2011.

6. Lee KW, Everett TH, Rahmutula D, et al: Pirfenidone prevents the development of a vulnerable substrate for atrial fibrillation in a canine model of heart failure. Circulation 114: 1703-1712, 2006.

7. Petrov VV, van Pelt JF, Vermeesch JR, et al: TGF-beta1-induced cardiac myofibroblasts are nonproliferating functional cells carrying DNA damages. Exp Cell Res 314: 1480-1494, 2008.

8. Shi Y and Massague J: Mechanisms of TGF-beta signaling from cell membrane to the nucleus. Cell 113: 685-700, 2003.
9. Schmierer B and Hill CS: TGFbeta-SMAD signal transduction: molecular specificity and functional flexibility. Nat Rev Mol Cell Biol 8: 970-982, 2007.

10. Chen P, Wu R, Zhu W, et al: Hypoxia preconditioned mesenchymal stem cells prevent cardiac fibroblast activation and collagen production via leptin. PLoS One 9: e103587, 2014.

11. Leask A: Potential therapeutic targets for cardiac fibrosis: TGFbeta, angiotensin, endothelin, CCN2, and PDGF, partners in fibroblast activation. Circ Res 106: 1675-1680, 2010.

12. Peng H, Carretero OA, Peterson EL and Rhaleb NE: Ac-SDKP inhibits transforming growth factor-betal-induced differentiation of human cardiac fibroblasts into myofibroblasts. Am J Physiol Heart Circ Physiol 298: H1357-H1364, 2010.

13. Lee MK, Pardoux C, Hall MC, et al: TGF-beta activates Erk MAP kinase signalling through direct phosphorylation of ShcA. EMBO J 26: 3957-3967, 2007.

14. Gao S,Liu Z,LiH,LittlePJ,Liu P and Xu S: Cardiovascular actions and therapeutic potential of tanshinone IIA. Atherosclerosis 220: 3-10, 2012.

15. Yang R, Liu A, Ma X, Li L, Su D and Liu J: Sodium tanshinone IIA sulfonate protects cardiomyocytes against oxidative stress-mediated apoptosis through inhibiting JNK activation. J Cardiovasc Pharmacol 51: 396-401, 2008.

16. Jiang FL, Leo S, Wang XG, et al: Effect of tanshinone IIA on cardiomyocyte hypertrophy and apoptosis in spontaneously hypertensive rats. Exp Ther Med 6: 1517-1521, 2013.

17. Xu S and Liu P: Tanshinone II-A: new perspectives for old remedies. Expert Opin Ther Pat 23: 149-153, 2013.

18. Yang L, Zou XJ, Gao X, et al: Sodium tanshinone IIA sulfonate attenuates angiotensin II-induced collagen type I expression in cardiac fibroblasts in vitro. Exp Mol Med 41: 508-516, 2009.

19. Wang $\mathrm{P}$, Zhou S, Xu L, et al: Hydrogen peroxide-mediated oxidative stress and collagen synthesis in cardiac fibroblasts: blockade by tanshinone IIA. J Ethnopharmacol 145: 152-161, 2013.

20. Tsai CT, Lai LP, Kuo KT, et al: Angiotensin II activates signal transducer and activators of transcription 3 via Racl in atrial myocytes and fibroblasts: implication for the therapeutic effect of statin in atrial structural remodeling. Circulation 117: 344-355, 2008.

21. Peng H, Carretero OA, Raij L, Yang F, Kapke A and Rhaleb NE: Antifibrotic effects of N-acetyl-seryl-aspartyl-Lysyl-proline on the heart and kidney in aldosterone-salt hypertensive rats. Hypertension 37: 794-800, 2001.

22. Zhang H, Pi R, Li R, et al: PPARbeta/delta activation inhibits angiotensin II-induced collagen type I expression in rat cardiac fibroblasts. Arch Biochem Biophys 460: 25-32, 2007.

23. Zhang DH, Cohn L, Ray P, Bottomly K and Ray A: Transcription factor GATA-3 is differentially expressed in murine Th1 and Th2 cells and controls Th2-specific expression of the interleukin-5 gene. J Biol Chem 272: 21597-21603, 1997.

24. Wang YS, Li SH, Guo J, et al: Role of miR-145 in cardiac myofibroblast differentiation. J Mol Cell Cardiol 66: 94-105, 2014.

25. Wang H, Leinwand LA and Anseth KS: Roles of transforming growth factor- $\beta 1$ and $\mathrm{OB}$-cadherin in porcine cardiac valve myofibroblast differentiation. FASEB J 28: 4551-4562, 2014.

26. Wu M, Han M, Li J, et al: 17beta-estradiol inhibits angiotensin II-induced cardiac myofibroblast differentiation. Eur J Pharmacol 616: 155-159, 2009.

27. Peng H, Carretero OA, Peterson EL, et al: N-Acetyl-serylaspartyl-lysyl-proline inhibits ET-1-induced collagen production by preserving Src homology 2 -containing protein tyrosine phosphatase-2 activity in cardiac fibroblasts. Pflugers Arch 464: 415-423, 2012

28. Liu X, Sun SQ, Hassid A and Ostrom RS: cAMP inhibits transforming growth factor-beta-stimulated collagen synthesis via inhibition of extracellular signal-regulated kinase 1/2 and Smad signaling in cardiac fibroblasts. Mol Pharmacol 70: 1992-2003, 2006.

29. Chen LX, Yang K, Sun M, et al: Fluorofenidone inhibits transforming growth factor-beta1-induced cardiac myofibroblast differentiation. Pharmazie 67: 452-456, 2012.

30. Wickramasinghe SR and Patel VV: Local innervation and atrial fibrillation. Circulation 128: 1566-1575, 2013.

31. Iwasaki YK, Nishida K, Kato T and Nattel S: Atrial fibrillation pathophysiology: implications for management. Circulation 124: 2264-2274, 2011.

32. Magnani JW, Rienstra M, Lin H, et al: Atrial fibrillation: current knowledge and future directions in epidemiology and genomics. Circulation 124: 1982-1993, 2011. 
33. Uesaka Y: Cerebral embolism and atrial fibrillation. Brain Nerve 65: 761-769, 2013 (In Japanese).

34. Burstein B, Libby E, Calderone A and Nattel S: Differential behaviors of atrial versus ventricular fibroblasts: a potential role for platelet-derived growth factor in atrial-ventricular remodeling differences. Circulation 117: 1630-1641, 2008.

35. Brown RD, Ambler SK, Mitchell MD and Long CS: The cardiac fibroblast: therapeutic target in myocardial remodeling and failure. Annu Rev Pharmacol Toxicol 45: 657-687, 2005.

36. Bosman FT and Stamenkovic I: Functional structure and composition of the extracellular matrix. J Pathol 200: 423-428, 2003.

37. Jugdutt BI: Remodeling of the myocardium and potential targets in the collagen degradation and synthesis pathways. Curr Drug Targets Cardiovasc Haematol Disord 3: 1-30, 2003.

38. Dobaczewski M, Chen W and Frangogiannis NG: Transforming growth factor (TGF)- $\beta$ signaling in cardiac remodeling. J Mo Cell Cardiol 51: 600-606, 2011.

39. Brooks WW and Conrad CH: Myocardial fibrosis in transforming growth factor beta(1)heterozygous mice. J Mol Cell Cardiol 32: $187-195,2000$.

40. Youn JY, Zhang J, Zhang Y, et al: Oxidative stress in atrial fibrillation: an emerging role of NADPH oxidase. J Mol Cell Cardiol 62: 72-79, 2013

41. Weber KT, Sun Y, Bhattacharya SK, Ahokas RA and Gerling IC: Myofibroblast-mediated mechanisms of pathological remodelling of the heart. Nat Rev Cardiol 10: 15-26, 2013.

42. Kong P, Christia P and Frangogiannis NG: The pathogenesis of cardiac fibrosis. Cell Mol Life Sci 71: 549-574, 2014.

43. Friedrichs K, Klinke A and Baldus S: Inflammatory pathways underlying atrial fibrillation. Trends Mol Med 17: 556-563, 2011

44. Tang W and Eisenbrand G: Salvia miltiorrhiza Bge. In: Chinese drugs of plant origin. Springer-Verlag, Berlin, pp891-902, 1992.
45. Yang L, Zou X, Liang Q, et al: Sodium tanshinone IIA sulfonate depresses angiotensin II-induced cardiomyocyte hypertrophy through MEK/ERK pathway. Exp Mol Med 39: 65-73, 2007.

46. Shan H, Li X, Pan Z, et al: Tanshinone IIA protects against sudden cardiac death induced by lethal arrhythmias via repression of microRNA-1. Br J Pharmacol 158: 1227-1235, 2009.

47. Yi SL, Liu XJ, Zhong JQ and Zhang Y: Role of caveolin-1 in atrial fibrillation as an anti-fibrotic signaling molecule in human atrial fibroblasts. PLoS One 9: e85144, 2014.

48. Rahmutula D, Marcus GM, Wilson EE, et al: Molecular basis of selective atrial fibrosis due to overexpression of transforming growth factor- $\beta 1$. Cardiovasc Res 99: 769-779, 2013.

49. Cucoranu I, Clempus R, Dikalova A, et al: NAD(P)H oxidase 4 mediates transforming growth factor-beta1-induced differentiation of cardiac fibroblasts into myofibroblasts. Circ Res 97: 900-907, 2005

50. Wei LH, Huang XR, Zhang Y, et al: Smad7 inhibits angiotensin II-induced hypertensive cardiac remodelling. Cardiovasc Res 99: 665-673, 2013.

51. Yue J and Mulder KM: Requirement of Ras/MAPK pathway activation by transforming growth factor beta for transforming growth factor beta 1 production in a Smad-dependent pathway. J Biol Chem 275: 30765-30773, 2000.

52. Leask A and Abraham DJ: TGF-beta signaling and the fibrotic response. FASEB J 18: 816-827, 2004.

53. de Caestecker MP, Parks WT, Frank CJ, et al: Smad2 transduces common signals from receptor serine-threonine and tyrosine kinases. Genes Dev 12: 1587-1592, 1998.

54. Rosenkranz S: TGF-beta1 and angiotensin networking in cardiac remodeling. Cardiovasc Res 63: 423-432, 2004. 